\title{
Data Governance Reference Model under the Lean Methodology for the Implementation of Successful Initiatives in the Peruvian Microfinance Sector
}

\author{
Alvaro Romero \\ Escuela de Ingeniería de Sistemas y \\ Computación \\ Universidad Peruana de Ciencias \\ Aplicadas (UPC) \\ Lima, Perú \\ u201316041@upc.edu.pe
}

\author{
Antony Gonzales \\ Escuela de Ingeniería de Sistemas y \\ Computación \\ Universidad Peruana de Ciencias \\ Aplicadas (UPC) \\ Lima, Perú \\ u201314811@upc.edu.pe
}

\author{
Carlos Raymundo \\ Escuela de Ingeniería de Sistemas y \\ Computación \\ Universidad Peruana de Ciencias \\ Aplicadas (UPC) \\ Lima, Perú \\ carlos.raymundo@upc.edu.pe
}

\begin{abstract}
Microfinance allows the integration of all sectors for the country's economic growth. Data duplicity, invalid data and the inability to have reliable data for decision-making are generated without a formal Governance. For this reason, Data Governance is the key to enable an autonomous, productive and reliable work environment for the use of these. Although Data Governance models already exist, in most cases they don't meet the requirements of the sector, which has its own characteristics, such as the volume exponential growth, data criticality, and regulatory frameworks to which it is exposed. The purpose of this research is to design a reference model for the microfinance organizations, supported by an evaluation tool that provides a diagnosis with the objective of implementing and improving the organization processes regarding Data Governance. This model was implemented based on the information of Peru's microfinance organizations, from which a 1.72 score was diagnosed, which is encouraging for the organization, since it shows that it has defined all its plans concerning Data Governance. Finally, after the validation, it was concluded that the model serves as a medium to identify the current status of these organizations to ensure the success of the Data Governance initiatives.
\end{abstract}

\section{CCS Concepts}

- Information systems $\rightarrow$ Enterprise applications

\section{Keywords}

Framework; Data Governance; Lean, Microfinance.

\section{INTRODUCTION}

Nowadays, microfinance are entities which provide financial services to a population with limited resources, which have been excluded from the traditional financial system. Organizations seek

Permission to make digital or hard copies of all or part of this work for personal or classroom use is granted without fee provided that copies are not made or distributed for profit or commercial advantage and that copies bear this notice and the full citation on the first page. Copyrights for components of this work owned by others than ACM must be honored. Abstracting with credit is permitted. To copy otherwise, or republish, to post on servers or to redistribute to lists, requires prior specific permission and/or a fee. Request permissions from Permissions@acm.org.

ICSIE 2019, April 9-12, 2019, Cairo, Egypt

(C) 2019 Association for Computing Machinery

ACM ISBN 978-1-4503-6105-7/19/04 ..\$15.00

https://doi.org/10.1145/3328833.3328859 to obtain competitive advantages through the management of their data, transforming them into clear and precise information [1], and microfinance organizations are no exception. The more data collected, the more likely organizations are to know the customer and processes. For this reason, there is a need to implement a Data governance model that meets the needs of the organization and provides a clear data management in the business.

According to a Digital Universe study, only $1 \%$ of the global data is analyzed and over $80 \%$ of the data is not protected [2]. In addition, IBM studies show that one of the most affected sectors by this gap of data governance is the financial sector, as the cost of each record of lost or stolen data is USD 141, which translates into a loss of USD 3.62 million per year [3]. Therefore, organizations must be able to assess their data management and have a clear management of data in business. However, only a few of the companies perform an analysis prior to the implementation of a Data Governance initiative. It is a problem, because the company will not know what they should really do, but the big question is how it is going to be done?, what methodology or model is the most appropriate?, if it's not implemented in a correct way it will have consequences in a short time period [4].

The objective of this research is to demonstrate the advantages and benefits of implementing Data Governance in an organization of the microfinance sector, in order to guarantee the integrity, availability and confidentiality of the data through a flexible proposal. In this sense, the araised proposal is the development of a Data Governance reference model for the microfinance organizations, which serves as a tool to perform the aforementioned evaluation. For this purpose, the proposal will be designed based on the Lean methodology, which helps detect activities that doesn't generate value in an organization. Thereby ensuring that all data in the organization meets the demands of the business and ensures the initiatives of Data Governance.

This document is divided into five sections. Section 2 contains information on the subject of research and review of the literature related to the topic presented in this document. Section 3 covers the developed proposal during the investigation period. Alternatively, the result of the validation of a model applied in a case study is found in Section 4. Finally, the conclusions of the investigation and the conclusions of the application of the model are presented in Section 5.

\section{STATE OF THE ARTH}

The Literature research was based on three approaches: models, services and data governance policies. Although research were 
conducted in order to study the financial market environment. In one of them, a CFO data governance structure practice was performed in UniCredit Bank AG [5]. As a result of the case study, the mission about data quality was incorporated into the storage team as a key factor among processes and final users.

The 3 best-known Data Governance Models were analyzed, which provide good practices for good control and use of the data. The Data Governance Institute (DGI) [4] is the oldest and best known source in the industry. Its use in companies has allowed data strategists, business stakeholders and IT leaders to work together to make decisions about how to manage data, obtain value, minimize costs and complexity. However, the model is designed to help large organizations that have more complex data or compliance environments. Likewise, this model is developed by several related items, such as programs, decision domain and components, which require a significant effort to learn and use. On the other hand, DAMA [6], which sees the Data Governance as a continuous improvement, taking into account the distinctive organizational and cultural problems. For this reason, this model defines 11 knowledge areas in the DAMA-DMBOK2. In this sense, this model requires the compliance of all its artifacts and is designed for large companies. Finally, there is IBM [7] which establishes 14 steps to implement Data Governance, of which ten are mandatory and four optional, along with associated IBM software tools and best practices to support an effective data governance program. This Process needs to be measured, and the results transmitted to the executive sponsors, on a regular basis.

In 2017, a research was initiated to demonstrate the advantages and benefits of the ability to objectively measure and evaluate the management performed in relation to the data of an organization in the microfinance sector, so as to guarantee integrity, availability and confidentiality of data through a flexible proposal [8]. In that same year, another investigation was made, due to the need to know the current technological and organizational situation that the organizations handle in relation to Data Governance, which will facilitate its implementation [9]. These investigations agree in the needs of microfinance organizations regarding Data Governance and highlight the importance of this topic, so they require its implementation and a method to improve its use. Especially in the microfinance sector to demonstrate and highlight why it is necessary to implement Data Governance in this scenario.

From 2012 onwards, some researchers reported about the importance of implementing Data Governance, as well as a research on this topic. The authors of these articles consider that in order to encourage companies to implement Data Governance, it is necessary to present successful stories in real companies [10] [11]. Therefore, they show different cases in different areas, as well as their benefits and drawbacks at the time of their implementation, providing a series of conclusions and recommendations about Data Governance in the studied sectors [12]. On one side, a study that explores the current literature on Data Governance and aims to offer a comprehensive analysis of the activities involved in data management. On the other side, they show the implementation of Data Governance Models under different technologies and scenarios [13]. All the cases focus on observing and highlighting, why the implementation of Data Governance is necessary [14].
Meanwhile, Data Governance policies in the financial sector consist of good data management through the use of models or good practices that contribute to better data management. In a 2009 research, a series of steps were defined, which will help the financial organizations to achieve a good data governance implementation [15]. With the purpose of showing that Data Governance should be used in the financial organizations in order to ensure definition, validity, consistency, quality, timeliness and availability of data owned. This study shows that there are policies that can affect the implementation of the Data Governance in the regulators to which the sector is subject to.

\section{CONTRIBUTION}

\subsection{Proposed model}

Microfinance have peculiar characteristics that have a direct impact on data management, based on the service provided to the client, the regulatory requirements and the financial system to which they are subject. There is a critical problem that characterizes the data manipulated by these organizations, which should be subject to what is indicated in the Law of Protection of personal data and whose consideration is a domain "Standards and compliance". This domain is crucial given the context of the DAMA model [10].

After the analysis, a model that allows the integration of the 5 domains is proposed, as shown in Figure 1, including the domain of "Standards and compliance" that refers to the requirements and norms to which companies in this sector are exposed. In this sense, the proposed model offers the organization the possibility of being evaluated in the microfinance sector in which it is governed, which allows it to use it as a point of reference to know its deficiencies with respect to the competition. In addition, this model is flexible and is based on the Lean methodology.

\subsubsection{Domains}

\subsubsection{Data governance scope}

This domain focus on identifying norms, compliance requirements, business rules related to data in the organization. Meaning, to be clear about what you want to achieve with the Data Governance program. As well as having the sponsorship of the main IT and business representatives to implement Data Governance, the way to obtain this sponsorship is to offer value in business terms

\subsubsection{Policies and responsibilities}

This domain groups a gradual level of practices related to the definition of policies and responsibilities for data governance in the organization. For that matter, we start from a poor level of practices which are characterized by the shortage of properly established and documented functions, until reaching a structured level of professionals forming a Data Governance Council in charge of ensuring the data governance in the organization

\subsubsection{Data governance organization structure}

This domain consists of establishing a plan to govern an organization's operations in order to ensure the authority to act in critical situations. Thus, the organizational structure is defined by 3 levels, strategic, tactical and operational level, as established by IBM [7]. 


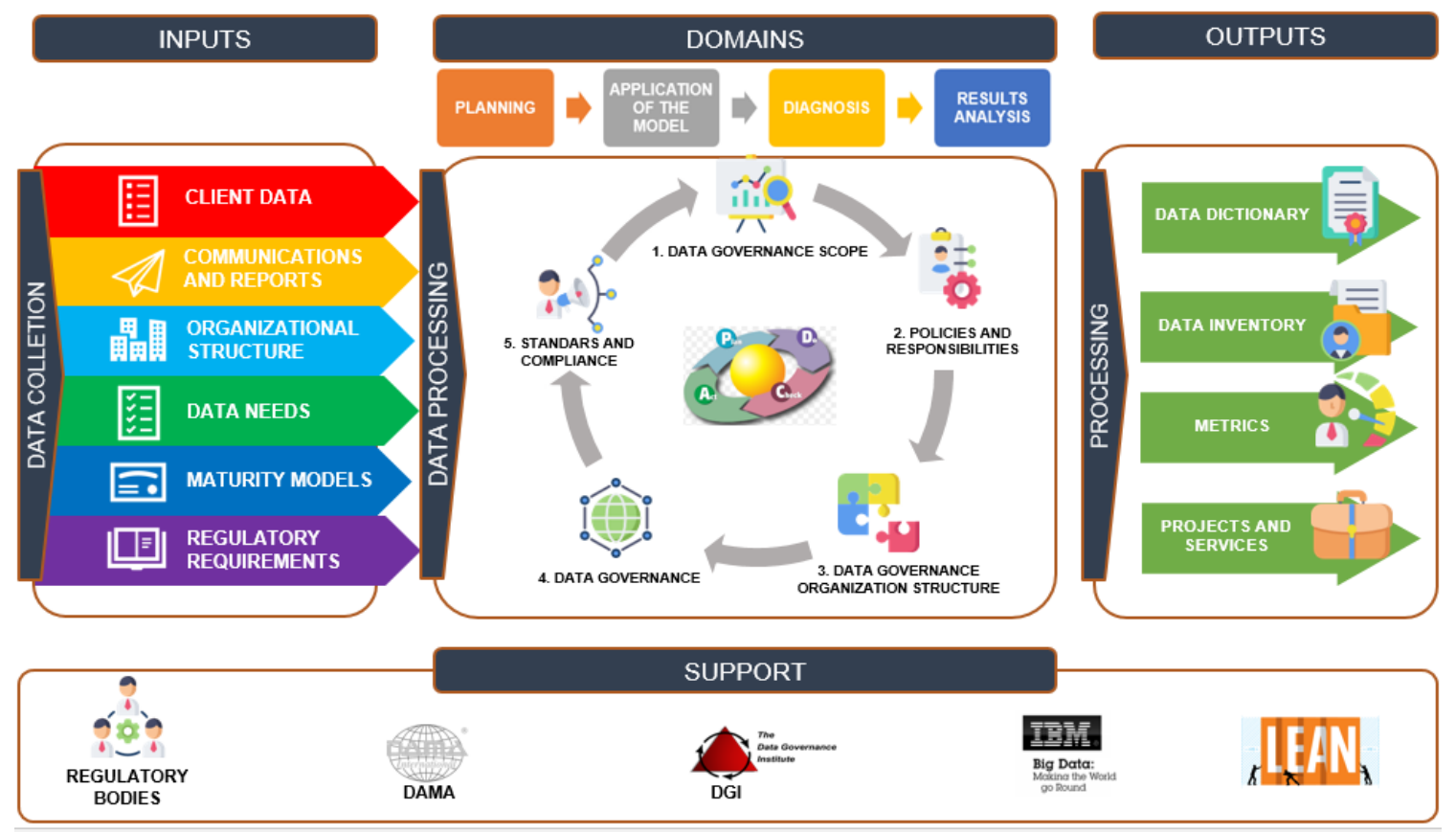

Figure 1. Data Governance Reference Model under the Lean methodology

\subsubsection{Data governance}

This domain importance lies in the management of processes and controls to guarantee data confidentiality, integrity and availability. The data is active because it has a commercial, tangible or intangible value.

\subsubsection{Standars and compliance.}

This domain highlights that every organization, regardless the sector they are in, is exposed to regulatory requirements. Where, some rules and guidelines indicate how the data should be managed. Part of this domain role is to monitor and guarantee regulatory compliance.

\subsection{Assessment proccess}

The proposed model offers the organization a possibility to be evaluated, allowing them to use it as a reference point to know its current status, through an Assessment as seen in Figure 2.

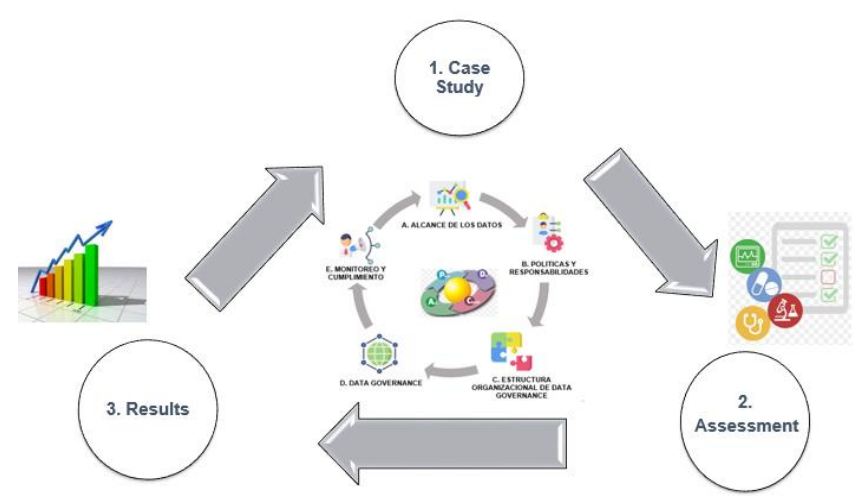

Figure 2. Assesment proccess

This assessment allows the interpretation and identification of the results to establish a road map for the improvement of the microfinance organization through the respective equations shown in Figure 3.
(1) Score $_{\text {Criteria }}=\frac{\sum_{k=1}^{k} w \cdot n}{k}$
Legend:
(2) Score $_{\text {Domain }}=\frac{\sum_{k_{1}=1}^{k_{1}} \text { Score }_{\text {Criteria }}}{k_{1}}$
w: Weight indicator of the implementation
(3) Score $_{\text {Model }}=\frac{\sum_{k_{2}=1}^{k_{2}} \text { Score }_{\text {Criteria }}}{k_{2}}$
$n:$ Maturity level
k: Quantity of evaluators
$k_{1:}$ Quantity of domain criteria
$k_{2}$ Quantity of model criteria

Figure 3. Score equation

Likewise, it was essential to consolidate these key areas, components or ideas into a single concept called evaluation criteria. As shown in Table 1.

Table 1. Evaluation Criteria Model

\begin{tabular}{|c|c|}
\hline Domain & Criteria \\
\hline \multirow{2}{*}{ Data Governance Scope } & Data Strategy \\
\hline & Data Role and Responsibility \\
\hline $\begin{array}{c}\text { Policies and } \\
\text { Responsibilities }\end{array}$ & Policies, procedures and methodology \\
\hline \multirow{2}{*}{$\begin{array}{l}\text { Data Governance } \\
\text { Organization Structure }\end{array}$} & Data committee Structure \\
\hline & Organization Culture \\
\hline \multirow{5}{*}{ Data Governance } & Data Definition \\
\hline & Data Inventory \\
\hline & Metadata \\
\hline & Data Acceptable Use \& Property \\
\hline & Data Dictionary \\
\hline \multirow{2}{*}{$\begin{array}{l}\text { Standards and } \\
\text { Compliance }\end{array}$} & Monitoring and Assessment \\
\hline & Internal \& External Auditing \\
\hline
\end{tabular}




\subsection{Levels}

The proposal regarding the model's level is based on the Deming Cycle, also known as the PDCA cycle. Each of these levels has been adapted to the data governance context in microfinance organizations. Table 2 shows the established levels of the model.

Table 2. Score Equivalences

\begin{tabular}{|c|c|}
\hline Score & Nivel \\
\hline 0 $>$ Score $<=\mathbf{1}$ & Plan \\
\hline 1 $>$ Score $<=\mathbf{2}$ & Do \\
\hline 2 $>$ Score $<=3$ & Check \\
\hline 3 $>$ Score $<=4$ & Act \\
\hline
\end{tabular}

\section{VALIDATION}

The validation process was implemented based on the following steps.

\subsection{Planning}

While planning the validation process, we addressed some fundamental aspects: validation scope, microfinance organizations and the case study work team. Regarding the validation scope, 5 domains of the model were applied. On the other hand, according to the case study, we worked with a microfinance company which we named the XYZ Company, in order to protect their data. This organization has 38 offices and is positioned as the fourth institution with the highest participation in consumer loans with a $6.9 \%$ within the small-size companies and microenterprise development entities, known as EDPYMEs in spanish. Finally, to complete the planning stage, it was necessary to select professionals with experience in the sector and data governance.

\subsection{Model application and diagnosis}

Once the planning stage is finished, the model application involves that each member of the team performs a corresponding evaluation in order to obtain the weighting of each criteria or domain of the proposed model. The purpose is to have the five perspectives and to be able to define an objective diagnosis for the organization. The diagnosis definition stage arises from the consolidation of the five evaluations in a single matrix allowing us to distinguish on which level of the PDCA cycle it is found, according to the domain. This consolidation is made based on the corresponding formulas to detect scores, as shown in Figure 3, that helped us notice that the level on which the organization is currently on is 1.72, meaning it is in Level Do.

$$
\begin{aligned}
\text { Score }_{\text {Model }} & =\frac{\sum_{k_{2}=1}^{k_{2}} \text { Score }_{\text {Criteria }}}{k_{2}} \\
\text { Score }_{\text {Model }} & =1.72
\end{aligned}
$$

Figure 4. Score Model Equation

\subsection{Results analysis}

In this stage we can identify the most relevant aspects, as well as the domain with the best performance and what it represents (See Figure 5). The biggest positive change compared to the general score is the Policies and Responsibilities domain, reaching the Level Check. On the other hand, the domain with the lowest score is Standards and Compliance, this means that the organization must focus on improving this aspect. Given these results, the organization must establish an action plan to improve their Data
Governance and the domains with a low level should have a high priority in short-term management.

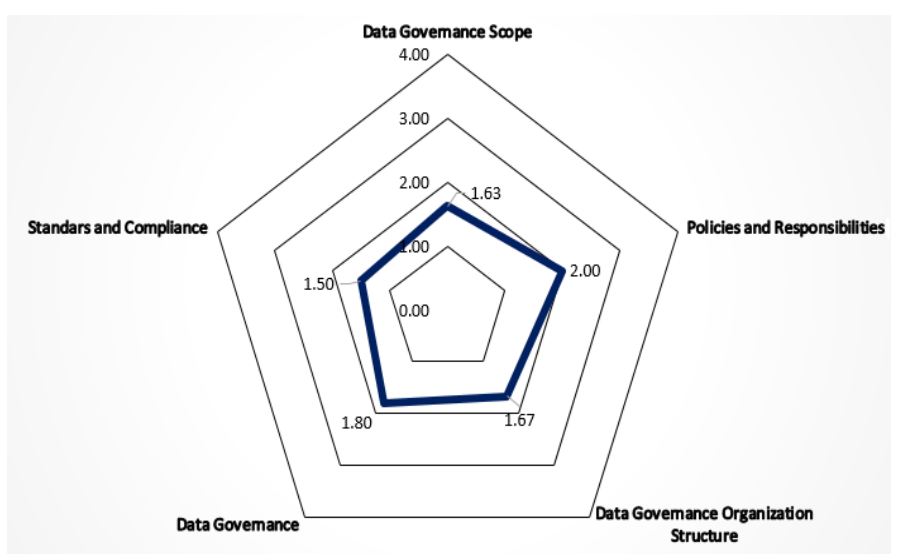

Figure 5. Representation of the score per domain.

\section{CONCLUSIONS}

The model was applied in the XYZ microfinance organization, where the 5 domains were evaluated. On that basis, a 1.72 score was obtained, positioning it in a Level Do. This level is encouraging for the organization, since it is considered that it has already defined all its plans regarding Data Governance. However, it is not considered a desired level because at this point the continuous process performance improvement is not yet sought, through incremental and innovative improvements in both processes and data level which supports the organization.

In this paper, the existing models of Data Governance were analyzed, identifying the best practices of each of them in order to propose a Data Governance model for the microfinance organizations, which includes 5 domains, 11 evaluation criterias, and 4 levels of continuous improvement, which have been addressed in the case study of the sector. Furthermore, the application of the model allowed the microfinance organization to evaluate its current status, in order to know its real management state and, subsequently, being able to establish priorities to manage an action plan regarding the diagnosis obtained.

\section{ACKNOWLEDGMENTS}

We will like to thank Ms. Lucero Valderrama and Mr. Julio Olivera for their help to validate this model, and Mr. Arturo Yep for his help with the interviewees.

\section{REFERENCES}

[1] NOVATICA. $2011 . \quad$ Ati.es. http://www.ati.es/novatica/2011/211/Nv211-Monografia.pdf

[2] The Guardian. 2012. "New Digital Universe Study Reveals Big Data Gap" http://www.emc.com/about/news/press/2012/201212-1101.htm

[3] Security, I. 2017. IBM 2017 Cost of Data Breach Study United States. https://www-03.ibm.com/security/uk-en/databreach/

[4] Thomas, G. 2017. The DGI Data Governance DGI Data Governance Framework.

[5] Traulsen S., Trobs M. 2011. Implementing Data Governance within a Financial Institution. Informatik schafft Communities. Jahrestagung der Gesellschaft für Informatik , 4.-7.10.2011, Berlin.

[6] 2017. Dama International, DAMA - DMBOK. (2017). 
[7] 2011. The IBM Data Governance Unified Process. Mc Pr Llc. (2011).

[8] Rivera, S., Loarte, N., Raymundo, C. and Dominguez, F. 2017. Data Governance Maturity Model for Micro Financial Organizations in Peru. Proceedings of the 19th. International Conference on Enterprise Information Systems.

[9] Ampuero, L., Alfaro, R., Raymundo, C. \& Mateos, F. 2017 Modelo de madurez Tecno-organizacional para la puesta en marcha exitosa de iniciativas de Data Governance. CICIC 2017 - Septima Conferencia Iberoamericana de Complejidad, Informatica y Cibernetica, Memorias pp. 93-99

[10] D. a. L. K. Wilbanks. 2012. «Data governance for SoS.,» International Journal of System of Systems Engineering, pp. 334-337.

[11] Putro, B., Surendro, K. and Herbert. 2016. Leadership and culture of data governance for the achievement of higher education goals (Case study: Indonesia University of Education)
[12] Thompson, N., Ravindran, R., \& Nicosia, S. 2015. Government data does not mean data governance: Lessons learned from a public sector application audit. Government Information Quarterly, 32(3), 316-322.

[13] Al-Ruithe, M., Mthunzi, S. and Benkhelifa, E. 2016. Data governance for security in IoT \& cloud converged environments. 2016 IEEE/ACS 13th International Conference of Computer Systems and Applications (AICCSA)

[14] Yulfitri, A. 2016. Modeling operational model of data governance in government: Case study: Government agency $\mathrm{X}$ in Jakarta. 2016 International Conference on Information Technology Systems and Innovation (ICITSI).

[15] Tarantino, A. 2009. Data Governance in Financial Risk Management. Risk Management in Finance: Six Sigma and Other Next-Generation Techniques. 